\title{
A COMPARISON BETWEEN THE EFFICACY OF TASK-BASED VS. DATA-BASED SEMG SONIFICATION DESIGNS
}

\author{
Daniel Verona
}

\author{
Texas A\&M University, \\ Department of Biomedical Engineering, \\ Emerging Technologies Building, 3120 TAMU, \\ 101 Bizzell Street, College Station, TX 77843 \\ daniel.j.verona@gmail.com
}

\begin{abstract}
Historically, many sonification designs that have been used for data analysis purposes have been based on data characteristics and have not been explicitly based on the listener's task. These sonification designs have often been described as annoying, confusing, or fatiguing. In the absence of a generally accepted theoretical framework for sonification design, there is a need for improvements in sonification design as well as a need for empirical evaluation of taskbased sonification designs. This research focuses on surface electromyography (sEMG) sonification and two sEMG data analysis tasks: determining which of two muscles contracts first and which of two muscles exhibits a higher exertion level. Both of these tasks were analyzed using a task analysis technique known as GOMS (Goals, Operators, Methods, Selection Rules) and two sonification designs were created based on the results of these task analyses. Two Data-based sEMG sonification designs were then taken from the sEMG sonification literature, and the four designs (2 Task-based and 2 Data-based) were empirically compared. Significant effects of sonification design on listener performance were found, with listeners scoring more accurately using the Task-based sonification designs. Based on these results, we argue for wider application of task analysis methods to sonification design and for the inclusion of task analysis methods into a generally accepted theoretical framework for sonification design.
\end{abstract}

\section{INTRODUCTION}

The auditory display community has known for quite some time that designing effective data sonifications is no small endeavor. A report published in 1999 highlighted the need for a sonification design method in order to establish a theoretical foundation upon which to base sonification designs [1]. Additionally, at the first ICAD conference in 1992, Sarah Bly referred to the lack of a theory of sonification as "a gaping hole impeding progress in the field" [2]. While strides have certainly been made since then towards establishing theories and guidelines for sonification design [3] [4] [5] [6] [7] [8] [9], a generally accepted sonification design framework, or theory of sonification design, still does not exist (S. Barrass, D. Brock, M. Gröhn, B. Walker, D. Worrall, personal communication, July 3, 2016).

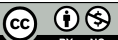

This work is licensed under Creative Commons Attribution - Non Commercial 4.0 International License.

The full terms of the License are available at

http://creativecommons.org/licenses/by-nc/4.0

https://doi.org/10.21785/icad2017.071

\author{
S. Camille Peres
}

\author{
Texas A\&M University, \\ Department of Environmental and Occupational \\ Health, School of Public Health \\ 212 Adriance Lab Rd., College Station, TX 77843 \\ peres@sph.tamu.edu
}

Arguably the most common type of sonification design in use today is parameter-mapping sonification, in which various parameters of sound (i.e. pitch, loudness, spatial location, timbre, etc.) are mapped onto data trends over a certain range and polarity. While this method of sonification can be effective for certain applications, it tends to result in sonifications that are annoying [10] [11] [12], confusing [13] [14], or fatiguing [15] [16]. Another problem with parametermapping sonification is that perceptual entanglement of various auditory parameters can lead to changes in one auditory dimension being perceived as changes in a different auditory dimension, which can obscure the meaning of a sonification [13]. This perceptual entanglement of various auditory parameters has led to what is known as The Mapping Problem, which is generally considered to be one of the primary obstacles currently facing sonification research [17].

Roddy and Furlong have proposed that improvements in sonification aesthetics could help to solve The Mapping Problem [18]. Sonification aesthetics have been understood in a variety of different ways over the years [9], and one important finding is that aesthetics and function cannot be treated independently in auditory display [10]. Building on this idea, Roddy and Furlong argue that sonification aesthetics deals primarily with meaning-making, and not with the overall "niceness" of the sound (which Roddy and Bridges refer to as sonification "cosmetics" [19]). Roddy and Furlong propose that looking to embodied cognition and creating sonifications that are mapped along embodied schemata may help listeners to derive the intended meaning from the sonification, and thus improve the aesthetic framework of the sonification. Embodied schemata are gestalt-like frameworks derived from the recurrent perceptual patterns encountered in daily life and they form the basic units of cognition. By mapping sonifications along these embodied dimensions, Roddy and Furlong argue that it could be possible to reconfigure entangled auditory dimensions into more comprehensible channels, thus circumventing The Mapping Problem and making sonifications that are more meaningful and easily understood [18]. The challenge they identified to this approach, however, is that the question of how to map specific embodied schemata to specific sonification tasks is not well understood.

Indeed, little research in general has been performed looking at how to design sonifications for specific tasks. This is due, in part, to the fact that many sonifications have not been explicitly designed to be tailored to the listener's task. Historically, many sonifications have been designed based on characteristics of the data being sonified (such as the type of data, number of data dimensions, number of data points etc.) [8], coupled with the designer's intuition [20] [21]. Additionally, many sonification designs to date have not been empirically evaluated [22], further compounding the 
problematic lack of understanding regarding how to design sonifications for specific tasks.

While a number of researchers have discussed the importance of considering the task when designing a sonification [3] [5] [6] [23] [24] [25] [26], few have discussed in significant depth how to go about understanding the listener's task using task analysis techniques (with the notable exception of Barrass's dissertation [3]). Seemingly fewer still have created sonification designs that were based on a task analysis, and to date, we are not aware of any empirical evaluation of task-analysis-based sonification designs.

One recent study investigated the effects of sonification design on listener performance for two different sEMG data evaluation tasks [27]. Results showed that one sonification design (Pitch/Loudness) yielded the most accurate listener performance for the first task (identifying which of two muscles contracted first), while a different design (Pitch/Loudness/Attack time) yielded the most accurate listener performance for the second task (identifying which of two muscles exhibited a higher exertion level).

Given this finding that sonification design efficacy was task-dependent, it seemed a natural progression to begin investigating task-specific sonification designs. This was done by using task analysis methods as a means for establishing sonification design criteria.

This paper presents the results of this investigation into task-based sonification designs which involved:

- Identifying an appropriate task analysis technique that would provide the needed information

- Performing task analyses of the two sEMG data evaluation tasks used in the study

- Creating two sonification designs based on the results of these task analyses

- Comparing these Task-based designs to two Databased designs taken from the sEMG sonification literature

\section{TASK ANALYSIS AND GOMS}

A task analysis is a design tool commonly used in fields such as Human Factors and Human Computer Interaction [28] [29]. Its purpose is to provide knowledge about users, their goals in accomplishing a task, their environment, the manual elements of the task, the cognitive elements of the task, the tools used to perform the task, the duration, order, and complexity of the task, as well as any other unique factors pertaining to the task [30].

The idea of using task analysis techniques to inform sonification design is not new, as previously mentioned. Barrass discussed the use of task analysis methods at length as part of his TaDa framework for sonification design [3]. Anderson also proposed the incorporation of task analysis methods into a framework for sonification design [5]. Walker and Nees stated that effective sonifications will require an understanding of the listener's function and goals within a system, that the task is a crucial consideration for the success or failure of a sonification, and that a display designer's knowledge of the task will constrain and inform the design of a sonification [6].

One issue we have not seen addressed in the sonification literature, however, is a discussion regarding what type of task analysis technique to use for informing sonification design. Many types of task analysis techniques have been developed, and each technique provides the designer with somewhat different information. One broad way to categorize the many different types of task analysis methods is to divide them into action-oriented methods and cognitive methods [31]. Action-oriented methods (such as the commonly used hierarchical task analysis, or HTA) focus on observable actions, or identifying, in top down fashion, the goal of the task, as well as the various subtasks and conditions under which those subtasks must be performed in order to achieve the goal. Cognitive methods, on the other hand, focus on analyzing and outlining the unseen mental processes diagnosis, decision making, problem solving, etc. - that can give rise to human error [31].

Based on this categorization, it at first seemed reasonable to take a cognitive approach to task analysis for use in informing sonification design, since comprehension of a sonification does not depend on observable actions, but rather on unseen mental operations. However, formal cognitive task analysis (CTA) methods may not be feasible for use in sonification design due to the fact that they typically require observation of expert performance, interviews with subject-matter-experts (SME's), or capturing an expert's performance with a think aloud protocol or subsequent recall [32]. Since interpreting a sonification is a primarily cognitive task, it would not be possible to visually observe an expert's performance of a sonification interpretation task to gain much useful information. To compound the problem, doing a think aloud protocol in real time for the interpretation of an EMG sonification would be difficult considering the interference speaking would have on listening to the sonifications.

To account for the cognitive aspects inherent to sonification interpretation, and avoid the ways in which CTA methods may not be ideal for decomposing sonification interpretation tasks, we are interested in the use of GOMS for informing sonification design. GOMS stands for Goals, Operators, Methods, and Selection Rules, and it is a form of HTA originally developed by Card, Moran, and Newell [33]. Its aim is to model and predict user behavior, or in the case of sonification, listener behavior. The four components of a GOMS model are as follows [34]:

- Goals: what the user is trying to accomplish. Goals can be, and often are, decomposed into Goal/Sub-goal hierarchies.

- Operators: actions performed in service of a goal. Operators can be perceptual, cognitive, or motor acts, or some combination of these.

- Methods: sequences of operators and sub-goal invocations that accomplish a goal.

- Selection Rules: when there is more than one method for accomplishing a goal, selection rules are the rules that the user employs to determine which method to use to accomplish the goal.

\section{APPLICATION OF GOMS TO IDENTIFY SONIFICATION DESIGN CRITERIA}

In this study, participants were asked to listen to sonifications of two channels of sEMG data, referred to as Muscle A and Muscle B, respectively. In the sonifications, both Muscle A and Muscle B began at rest, contracted at close to the same time, remained contracted for a few seconds, and then returned to rest. After listening to each sonification, participants were asked to perform the following two sEMG data evaluation tasks: 


\begin{tabular}{|c|}
\hline Goal: DETERMINE IF A OR B CONTRACTS FIRST, OR \\
IF THEY CoNTRACT SIMULTANEOUSLY \\
Method for TIME Goal: \\
SG 1. Start Task \\
SG 2. Identify $1^{\text {st }}$ Muscle Activation \\
SG 3. Determine if $1^{\text {st }}$ Activation was Muscle A \\
or Muscle B \\
SG 4. Determine if other Muscle Activated \\
also \\
SG 5. If Unsure regarding Subgoal 3, Identify \\
SG 6. Determine if $2^{\text {nd }}$ Activation was A or B \\
SG 7. Determine if A or B Contracted First \\
SG 8. Report if A or B Activated First
\end{tabular}

\begin{tabular}{|c|c|}
\hline \multicolumn{2}{|c|}{ Method for Subgoal 1: } \\
Start Task \\
Op 1. Grasp computer mouse \\
op 2. Point with mouse to PLAY button \\
op 3. Left-click PLAY button \\
\hline
\end{tabular}

\section{Method for Subgoal 2:} Identify $1^{\text {st }}$ Muscle Activation

op 1. Perceive sonic event indicating muscle activation

op 2. Place sonic event in auditory store

op 3. Shift attention to auditory store

\section{Method for Subgoal 3:}

Determine if $1^{\text {st }}$ Activation was $A$ or $B$

op 1. Perceive unique sonic identifier for A or B

op 2. Equate sonic identifier with A or B

op 3. Place identification of A or B into working memory

\section{Method for Subgoal 4:}

Determine if other Muscle Activated also

Op 1. Sonic event indicating other muscle activating simultaneously perceived?

op 2. If yes, store this knowledge in working memory

Op 3. If no, then keep identification of $A$ or B (from Subgoal 3) in working memory

\section{Method for subgoal 5 :}

If Unsure regarding Subgoal 3, Identify $2^{\text {nd }}$ Muscle Activation

Same as Method for Subgoal 2, but for second muscle activation

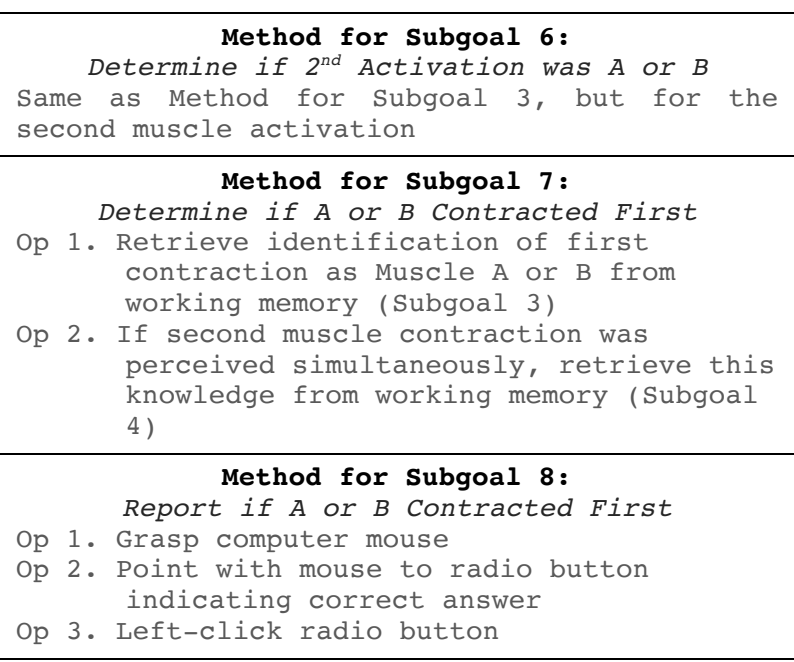

Goal: DETERMINE IF A OR B HAS A HIGHER EXERTION LEVEL, OR IF THEY ARE THE SAME

Method for LEVEL Goal:

SG 1. Start Task

SG 2. Identify Muscle $A^{\prime}$ s Activation

SG 3. Identify Muscle B's Activation

SG 4. Monitor A's Exertion Relative to B'S Exertion during muscle contraction

SG 5. Identify when A Returns to Rest

SG 6. Identify when B Returns to Rest

SG 7. Determine if A or B had Higher Exertion Level

SG 8. Report if A or B had Higher Exertion Level

\section{Method for Subgoal 1:}

Start Task

Op 1. Grasp computer mouse

Op 2. Point with mouse to PLAY button

Op 3. Left-click PLAY button

\section{Method for Subgoal 2:}

Identify Muscle A's Activation

op 1. Perceive sonic event

op 2. Perceive unique sonic identifier for Muscle A

op 3. Place sonic event in auditory store

Op 4. Shift attention to auditory store

Op 5. Equate identifier with Muscle A

Method for subgoal 3 :

Identify Muscle $B^{\prime} s$ Activation

Same as Method for Subgoal 2, but for Muscle $\mathrm{B}$

\section{Method for Subgoal 4:}

Monitor A's Exertion Relative to B's Exertion

op 1. Use echoic memory to continuously update $A^{\prime}$ 's max exertion

Op 2. Use echoic memory to continuously update $B^{\prime} \mathrm{s}$ max exertion

Op 3. Place max exertion in working memory

\section{Method for Subgoal 5:}

Identify when A Returns to Rest

op 1. Perceive sonic event indicating Muscle A returning to rest

op 2. Place sonic event in auditory store

op 3. Shift attention to auditory store

op 4. Stop continuously updating max exertion for Muscle A

\section{Method for Subgoal 6:}

Identify when $B$ Returns to Rest

Same as Method for Subgoal 5, but for Muscle B

\section{Method for Subgoal 7 :}

Determine if $A$ or $B$ had $a$ Higher Exertion Level

Op 1. Retrieve max exertion level from working memory

Op 2. Equate max exertion level with Muscle A or Muscle B

\section{Method for Subgoal 8:}

Report if $A$ or $B$ had a Higher Exertion Level

Op 1. Grasp computer mouse

op 2. Point with mouse to radio button indicating correct answer

Op 3. Left-click radio button

Figure 1 - The left column shows the GOMS analysis for the TIME task (determining which of two muscles contracts first), and the right column shows the GOMS analysis for the LEVEL task (determining which of two muscles exhibits a higher exertion level). In these graphs, "SG" stands for 'Subgoal' and "Op” stands for 'Operator'. 
1. TIME Task: Identify which muscle (A or B) contracted first

2. LEVEL Task: Identify which muscle (A or B) exhibited a higher exertion level

To design sonifications specifically for these two tasks, GOMS analyses were performed for both tasks, and the results are shown above in Figure 1. These GOMS analyses only show Goals, Subgoals, and Operators. The Method is to follow the Subgoals in numerical order, and for each Subgoal to follow the Operators in numerical order. The assumption is that there are not additional Methods that would allow for the accomplishment of each Goal, and thus there are no Selection Rules shown for selecting between competing Methods. Identification of the various Subgoals involved for each task served as the primary factor in establishing sonification design criteria for each task.

\subsection{Design criteria for the TIME task}

For the task of identifying which muscle contracts first, the analysis in the left column of Figure 1 indicates that a listener must be able to understand that the task has started (Subgoal 1 ), identify when the first muscle changes state from rest to contraction (Subgoal 2), then determine if that muscle was Muscle A or Muscle B (Subgoal 3). If the sonification does not give the listener the ability to accomplish even one of these Subgoals, the listener will not be able to complete the task. Thus, the design criteria for the sonifications based on the GOMS model for the TIME task are:

1. The start of the listening task must be evident

2. The sound of the first muscle changing state from rest to contraction must be evident

3. The listener must have a way of distinguishing between the sound of Muscle A activating and the sound of Muscle B activating

\subsection{Design criteria for the LEVEL task}

For the task of identifying which muscle has a higher exertion level, the analysis shown in the right column of Figure 1 indicates that the listener must be able to understand that the task has started (Subgoal 1), determine when both muscles change state from rest to contraction (Subgoals 2 and 3), monitor the exertion level difference between Muscle $\mathrm{A}$ and $\mathrm{B}$ for the duration of their contractions (Subgoal 4), identify when both muscles revert back to rest (Subgoals 5 and 6), then determine if Muscle $\mathrm{A}$ or $\mathrm{B}$ had a higher exertion level (Subgoal 7). Once again, failure to accomplish any of these Subgoals will prevent the listener from completing the task. Thus, the design criteria for the sonifications based on the GOMS model for the LEVEL task are:

1. The start of the listening task must be evident

2. The sound of both muscles changing state from rest to contraction must be evident

3. The exertion level difference between the two muscles must be evident

4. The sound of both muscles changing state from contraction back to rest must be evident

\section{METHODS}

Each sonification design created for this study was coded in the SuperCollider audio synthesis environment. All sEMG data processing (rectifying and filtering) was performed using MATLAB.

\subsection{Study design}

This study compared the efficacy of two Task-based sonification designs to two Data-based sonification designs taken from the EMG sonification literature, for two different tasks - muscle activation time and muscle exertion level. There were thus three main independent variables (IVs: Databased design, Task-based design, and Task) with two levels of each variable. Further, there were 4 levels of difficulty for each task, adding another IV. For the Data-based designs, the first level was a pitch mapping and the second level was a loudness/timbre mapping, referred to henceforth as a loudness mapping. These designs were taken from a 2012 study investigating sonification of EMG data for use in analyzing human movements [35]. The details of these designs are explained below in section 4.2. For the Taskbased designs, the first level was the "Task-Panning" design which used short beeps to indicate the onset of muscle activation and a panning tone to indicate exertion level difference. The second level was the "Task-Filter" design which also used short beeps to indicate the onset of muscle activation, but used a panned filter cutoff mapping to indicate muscle exertion difference. There were two dependent variables associated with the two levels of the task IV to assess performance for each design: judgment of muscle activation time (TIME task) and judgment of muscle exertion level (LEVEL task). The IV's and Levels are described in Table 1 below.

\begin{tabular}{|c|c|c|}
\hline \multicolumn{3}{|c|}{$\begin{array}{l}\text { Table 1: IV and Level for the four sonification designs and } \\
\text { two tasks. }\end{array}$} \\
\hline $\begin{array}{l}\text { IV 1: Data- } \\
\text { based }\end{array}$ & IV 2: Task-based & IV 3: Task \\
\hline Data-Pitch & Task-Panning & $\begin{array}{c}\text { Muscle } \\
\text { activation time } \\
\text { difference }\end{array}$ \\
\hline Data-Loudness & Task-Filter & $\begin{array}{c}\text { Muscle } \\
\text { exertion level } \\
\text { difference }\end{array}$ \\
\hline
\end{tabular}

This study was a within-subjects factorial design. Participants listened to 16 sonifications with each of the four designs for a total of 64 sonifications. The presentation order of the four sonification designs was counterbalanced to account for training effects.

\subsection{Data-based designs}

As previously mentioned, the two Data-based designs used in this study were taken from a 2012 paper by Matsubara et al. [35]. We chose to use designs from Matsubara's paper because participants in Matsubara's study were asked to perform sEMG data evaluation tasks that were similar to the sEMG data evaluation tasks that we asked our participants to perform. There were three design methods used in Matsubara's study: Method A: Pitch, Method B: Loudness/Polyphonic Timbre, and Method C: Timbre. Methods A and B were chosen as the Data-based designs for 
this study because they resulted in the best listener performance during Matsubara's study.

The Data-Pitch design was created according to the specifications laid out in Matsubara [35] for Method A, with the first channel of sEMG data (Muscle A) sonified using a sine wave tone over a frequency range of $300-525 \mathrm{~Hz}$, and the second channel of sEMG data (Muscle B) sonified using a sine wave tone over a lower frequency range of 165-345 $\mathrm{Hz}$. Additionally, we decided to spatialize this design by panning the first channel of sEMG data (A) hard left and panning the second channel of sEMG data (B) hard right. We made this decision based on our previous findings that spatialization helps listeners distinguish between sEMG channels [27].

The Data-Loudness design was also created according to the specifications laid out in Matsubara's paper for Method B. Again, we spatialized the design in an attempt to enhance listener performance in keeping with our previous findings.

\subsection{Task-based designs}

The Task-Panning design was based on the design criteria for the TIME and LEVEL tasks from the task analyses. To ensure that the listener would know that the sonification was playing, a soft, low-pass-filtered white noise was played while the muscles were at rest. The cutoff frequency of the LPF was set to $1000 \mathrm{~Hz}$.

To indicate when each muscle activated, short beep tones were played when each muscle began to contract. To indicate the contraction of Muscle A, a short beep ( $0.07 \mathrm{sec}$ duration) using a triangle wave at a frequency of $440 \mathrm{~Hz}$ was played in the left ear. To indicate the contraction of Muscle B, a short beep of equal duration using a triangle wave at a frequency of $330 \mathrm{~Hz}$ was played in the right ear. Once both muscles had begun to contract, the LPF white noise was turned off and a tone indicating exertion level difference began to play.

To indicate the exertion level difference between Muscle $\mathrm{A}$ and $\mathrm{B}$, the sonification code calculated the difference in amplitude between $\mathrm{A}$ and $\mathrm{B}\left(\mathrm{Amp}_{\mathrm{A}}-\mathrm{Amp}_{\mathrm{B}}\right)$, and then mapped this difference to the pan position of a tone that played during muscle contraction. If the difference was positive, this meant that Muscle A had a higher exertion level and the tone panned left, and vice versa. When the difference in exertion was small $(\sim 0.05 \mathrm{~V})$, the tone panned slightly left or right (to a value of $+/-0.7$ on SuperCollider's Pan2 function). When the difference in exertion was larger $(>0.1$ $\mathrm{V})$, the tone panned hard left or right.

After muscle contraction, the tone became silent and the white noise returned to indicate that the muscles had returned to rest.

The Task-Filter design was also based on the design criteria for the TIME and LEVEL tasks from the task analyses. For this design, when the muscles were at rest, a soft, low-pass-filtered sawtooth wave was played, one in the left channel to represent Muscle A and one in the right channel to represent Muscle B. The frequency of the waves was $100 \mathrm{~Hz}$, and the cutoff frequency of the LPF was 300 $\mathrm{Hz}$. The two waves were played at equal amplitude so as to be perceived in the center of the stereo field.

To indicate when each muscle activated, short beep tones were played right when each muscle began to contract. To indicate the contraction of Muscle A, a short beep ( $0.09 \mathrm{sec}$ duration) using an additive synthesis tone with a fundamental frequency of $300 \mathrm{~Hz}$ was played in the left ear. To indicate the contraction of Muscle B, the same short beep was played in the right ear. The fundamental frequency of $300 \mathrm{~Hz}$ was chosen so that these beeps would "sit on top of" the sawtooth wave tones (which were LPF'd at $300 \mathrm{~Hz}$ ) and not interfere with them.

To indicate the exertion level difference between Muscle $\mathrm{A}$ and $\mathrm{B}$, the sonification code calculated the amplitude difference in the same manner as in the Task-Panning design. If the difference was positive, this meant that Muscle A (in the left channel) had a higher exertion level and the difference was mapped to the cutoff frequency of the LPF in the left channel, such that the cutoff increased to allow more high frequency content to be heard in the left channel during muscle contraction. The opposite occurred when the amplitude difference was negative, with the cutoff of the right channel's LPF increasing to indicate that Muscle B had a higher exertion level. For small exertion differences $(0.05$ $\mathrm{V}$ ), the cutoff would increase from $300 \mathrm{~Hz}$ to $1200 \mathrm{~Hz}$, and for larger exertion differences $(0.15 \mathrm{~V})$, the cutoff increased from $300 \mathrm{~Hz}$ to $3600 \mathrm{~Hz}$.

After muscle contraction, the cutoff of both LPF's was set back to $300 \mathrm{~Hz}$ to indicate that the muscles had returned to rest.

\subsection{Activation time/Exertion level differences}

For each of the four sonification designs, participants listened to 16 sonifications. Of these 16,4 displayed both muscles activating at the same time, and 4 each displayed both muscles activating $0.13 \mathrm{sec}$ apart, $0.26 \mathrm{sec}$ apart, and $0.39 \mathrm{sec}$ apart.

Additionally, out of the 16, 4 sonifications displayed both muscles exhibiting the same exertion level, and 4 each displayed both muscles exhibiting a $0.05 \mathrm{~V}, 0.10 \mathrm{~V}$, and 0.15 $\mathrm{V}$ amplitude difference during muscle contraction. The 16 sonifications for each design were numbered according to Table 2 below.

\begin{tabular}{|c|c|c|c|c|c|}
\hline & & \multicolumn{4}{|c|}{ Activation time difference } \\
\hline & & $0 \mathrm{sec}$ & $\begin{array}{c}0.13 \\
\mathrm{sec} \\
\end{array}$ & $\begin{array}{c}0.26 \\
\mathrm{sec} \\
\end{array}$ & $\begin{array}{c}0.39 \\
\mathrm{sec} \\
\end{array}$ \\
\hline \multirow{4}{*}{$\begin{array}{l}\text { Exertion } \\
\text { level } \\
\text { difference }\end{array}$} & $0 \mathrm{~V}$ & 1 & 2 & 3 & 4 \\
\hline & $0.05 \mathrm{~V}$ & 5 & 6 & 7 & 8 \\
\hline & $0.10 \mathrm{~V}$ & 9 & 10 & 11 & 12 \\
\hline & $0.15 \mathrm{~V}$ & 13 & 14 & 15 & 16 \\
\hline
\end{tabular}

As an example, Sonification \#1 for any given design displayed both muscles contracting at the same time $(0 \mathrm{sec}$ activation time difference) and exhibiting the same exertion level (0 $\mathrm{V}$ amplitude difference during contraction). Similarly, Sonification \#11 in any given design displayed a $0.26 \mathrm{sec}$ difference between the activation of Muscle A and the activation of Muscle $\mathrm{B}$, and a $0.1 \mathrm{~V}$ difference in amplitude between Muscle A and Muscle B. The order in which each sonification within a given design was presented was randomized for each counterbalance.

\subsection{Participants}

Forty students and faculty from Texas A\&M university participated in this study (27 male, 16 female, ages 19-59). They all self-reported as not having any hearing impairment that would interfere with their ability to participate. At the beginning of each session, participants signed a consent form, completed a demographic survey, and were asked 
about their knowledge of and experience with EMG data. After this, they were briefly trained on what sEMG data is, what sonification is, and how sEMG data can be sonified.

\subsection{Computer/Audio setup}

The study was run locally through a browser (Google Chrome) using the XAMPP environment in conjunction with a MySQL database for recording participant responses. Participants listened to the sonifications through a pair of Beyerdynamic DT 770 Pro headphones.

\subsection{Measures}

Listener accuracy was measured as a proportion of correct responses for both tasks. After listening to each sonification, participants were asked two multiple choice questions, one each for the TIME and LEVEL tasks. The choices were:

1. Muscle A activated first (or had a higher exertion)

2. Muscle B activated first (or had a higher exertion)

3. A and B had the same activation time (or exertion level)

4. Unsure

For example, if a listener correctly identified if Muscle A or B contracted first for 8 out of the 16 Data-Pitch sonifications, their score was $8 / 16=0.5$ for that Design/Task pair.

\section{RESULTS}

\subsection{Overall performance}

As seen in Figure 2, there was no effect of Task, $F(1,42)=$ $1.782, p=0.189$. However, there was a main effect of design, $\mathrm{F}(2.079,87.29)=91.23, \mathrm{p}<0.001$, eta squared 0.69 , and an interaction between Task and Design $F(2.55,107.23)=$ $32.83, \mathrm{p}<0.001$, eta squared $=0.44$.

Bonferroni pairwise comparisons indicated that performance was different based on design with the DataPitch design having the worst performance and Task-Filter having the best $(\mathrm{p}<0.001)$. Data-Loudness and TaskPanning had performance levels in between those two and Bonferroni pairwise comparisons show that performance on all designs were significantly different from each other (all p's $<0.001$ ). As shown in Figure 1, there was an interaction between Design and Task with the Data-Pitch design having better performance for the TIME task $(p<0.034)$, and the Task-Filter design having better performance for the LEVEL task $(\mathrm{p}<0.028)$.

\subsection{Performance by difficulty level}

Figure 3 shows the results by difficulty level for the activation time task. This figure shows that performance differed by Design with the Task-based designs resulting in better performance than the Data-based designs (all p's < 0.01). The Task-based designs and Data-based designs were not different from each other ( $\mathrm{p}$ 's $>0.29), \mathrm{F}(2.246,94.318)=$ $19.60, p<0.001$, eta squared $=0.318$. Figure 2 also shows that there were overall differences in performance based on the Activation Time Differences (ATD) with better performance when the differences

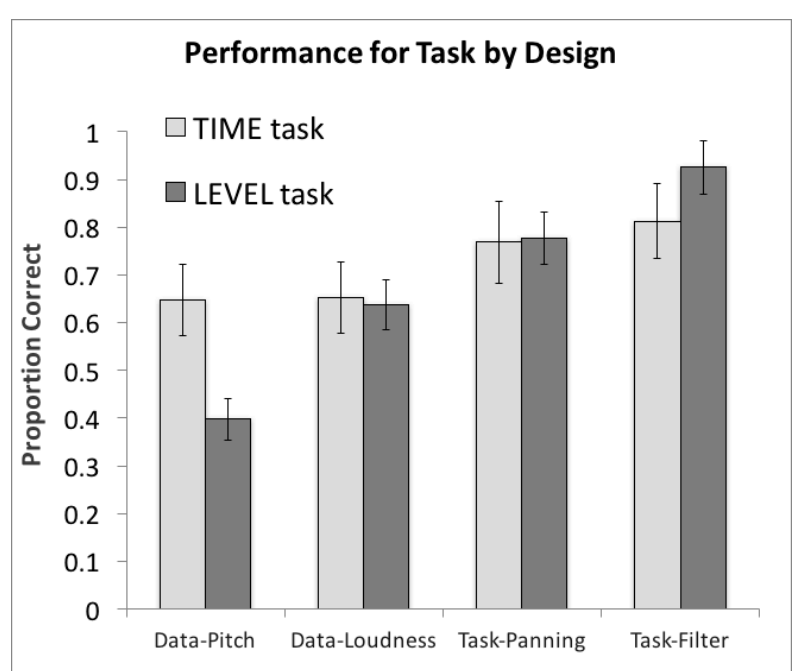

Figure 2 - Overall listener performance for each Design and for both Tasks

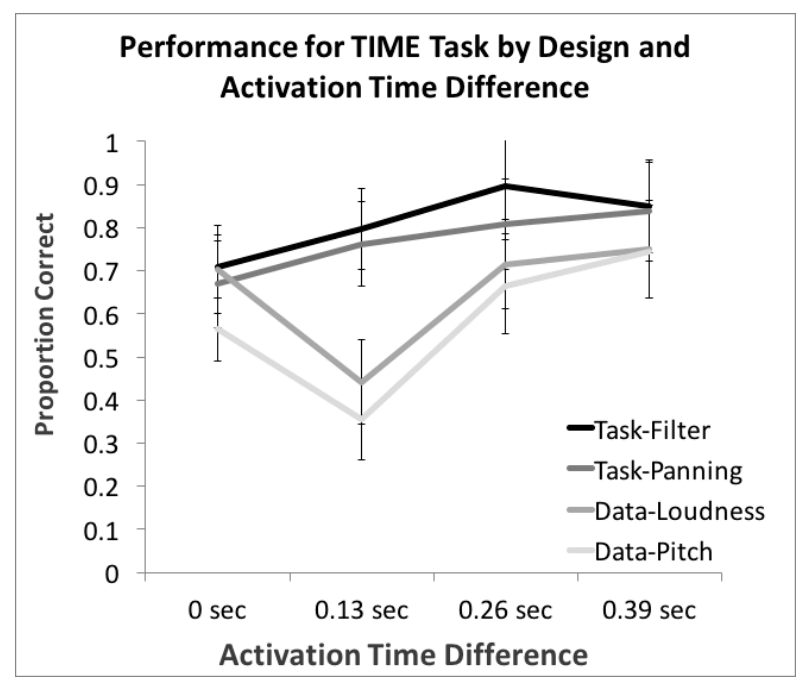

Figure 3 - Listener performance for the TIME Task for each Design and Activation Time Difference (ATD). ATD = time difference between activation of Muscle A and Muscle B

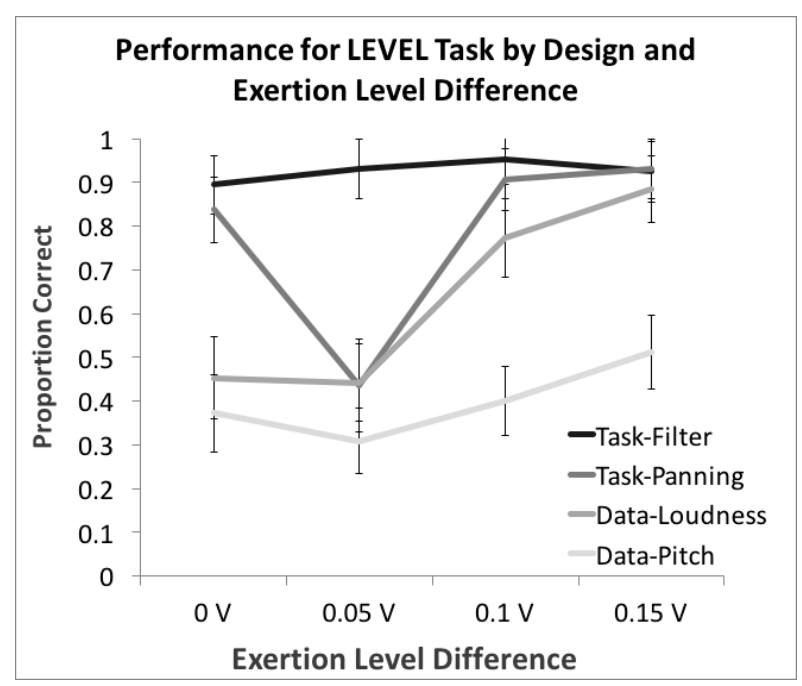

Figure 4 - Listener performance for the LEVEL task for each Design and Exertion Level Difference (ELD). ELD = amplitude difference during contraction between Muscle A and Muscle B 
were larger $(0.26 \mathrm{sec}$ and $0.39 \mathrm{sec})$ (all p's < 0.001), F(1.539, $64.65)=12.27, \mathrm{p}<0.001$, eta squared $=0.23$. The differences by Level differed by Design for the TIME task with Bonferroni comparisons indicating that Data-Pitch (0.13 sec) was different than all others and Data-Pitch $(0 \mathrm{sec})$ was different than Data-Pitch $(0.39 \mathrm{sec})$; Data-Loudness $(0.13$ sec) was different than all others; Task-Panning showed no performance differences by level; and Task-Filter $(0.13 \mathrm{sec})$ was different than Task-Filter $(0.26 \mathrm{sec}), \mathrm{F}(5.43,228.11)=$ $7.68, \mathrm{p}<0.001$, eta squared $=0.12$.

Figure 4 shows that overall performance for the LEVEL task differed by Design with the Task-Filter Design (all p's $<$ 0.01 ) resulting in the best performance and Data-Pitch design resulting in the worst. Bonferroni comparisons showed that all designs were different from each other with performance on the Task-Panning being lower than Task-Filter and greater than Data-Loudness, $F(2.37,99.55)=154.54, p<0.001$, eta squared $=0.79$. Figure 4 also shows that there were differences in performance based on the Exertion Level Differences with performance generally increasing as exertion level differences increased (all p's $<0.038$ ). The exception to this was the decrease in performance from $0 \mathrm{~V}$ difference to $0.05 \mathrm{~V}$ difference, $\mathrm{F}(1.95,81.91)=38.12$, $\mathrm{p}<$ 0.001 , eta squared $=0.476$. The differences by Level differed by Design with Bonferroni comparisons indicating that DataPitch $(0.05 \mathrm{~V})$ was different than Data-Pitch $(0.15 \mathrm{~V})$, DataLoudness $(0.15 \mathrm{~V})$ was different than Data-Loudness (0, $0.05,0.1 \mathrm{~V})$, Data-Loudness $(0,0.05 \mathrm{~V})$ was different than Data-Loudness $(0.1,0.15 \mathrm{~V})$, Task-Panning $(0.05 \mathrm{~V})$ was different than Task-Panning $(0,0.1,0.15 \mathrm{~V})$, and Task-Filter showed no differences between levels, $\mathrm{F}(5.563,233.648)=$ $15.534, \mathrm{p}<0.001$, eta squared $=0.27$.

\section{DISCUSSION}

The results of this study clearly indicate that for interpreting sEMG sonifications for these tasks, using sonification designs based on the task results in superior performance, particularly for the TIME task.

For the TIME task, The Data-Pitch and Data-Loudness designs showed poor performance when the activation time difference was $0.13 \mathrm{sec}$. By contrast, the Task-Panning and Task-Filter designs showed performance that essentially increased as the TIME difference increased (Figure 3). This was likely due to the fact that the Task-based designs were designed specifically to create a large, temporally precise contrast between the sound of a muscle at rest and the sound of a muscle beginning to contract. The Data-based designs did not provide the same level of perceivable contrast between the sound of a muscle changing state from rest to activation.

For the LEVEL task, there were interesting interactions based on the difficulty level of task with the more difficult stimuli $(.05 \mathrm{~V})$ reducing performance remarkably more with the Task-Panning design than any of other of the designs. Further, there were differences in performance between the Data-Pitch and Data-Loudness designs for the LEVEL task. This is likely due to two things: the Data-Pitch design used different pitch ranges for Muscles A and B which made a direct comparison between the two difficult, and the DataLoudness design essentially made use of a panning effect by mapping muscle exertion level to loudness. Since the designs were spatialized into left and right audio channels, at larger exertion level differences $(0.1$ and $0.15 \mathrm{~V})$, the DataLoudness design acted like a panning mapping, and indeed, the Data-Loudness design showed similar performance for the LEVEL task as both of the Task-based designs, which both made explicit use of panning (see Figure 4).

These findings that Task-based designs can result in better listener performance than Data-based designs strongly suggest the broader integration of task-based approaches into the sonification design problem space. Additionally, they indicate that the inclusion of task analyses within a theoretical framework for sonification design may facilitate the development of this illusive framework.

Task-based approaches to sonification design do not seem to be well represented in the auditory display literature. It is not uncommon in the EMG sonification literature, for example, to see an explanation for how a sonification was designed but to not see an explanation for why it was designed that way. Justifications for design decisions are sometimes given, but they rarely seem to go beyond appeals to sonic cosmetics or "traditional" mappings like pitch and loudness.

A task-based approach to sonification design could allow sonification designers to use Human Factors and HCI design methodology to identify sonification design criteria. In so doing, this approach could afford sonification designers stronger justification for design decisions, as well as facilitate easier communication between sonification designers and $\mathrm{HF} / \mathrm{HCI}$ researchers - which could broaden the ICAD community's impact and stimulate wider interest in the field. As mentioned in the introduction to this paper, Roddy and Furlong have discussed sonification aesthetics and the problem of a disembodied approach to sonification design. They have argued that leveraging knowledge of embodied cognition and embodied schemata may help sonification designers to circumvent the Mapping Problem by mapping sonifications along embodied dimensions. Task-based approaches to sonification design may not be embodied in and of themselves, but since task analyses can provide indepth knowledge of a user's task, and since mapping sonifications along embodied dimensions requires a deep understanding of the user's task, it seems that task-based approaches to sonification design may aid in identifying useful embodied schemata along which to map sonifications for specific tasks.

In conclusion, task analysis techniques are well established in fields such as Human Factors and HCI, where design decisions are critical. In this study, implementing task analysis techniques into the design of auditory displays was shown to be an effective approach for creating interpretable sonifications. Further use of task analysis techniques in auditory display is thus recommended. This study has served as a "proof-of-concept," and we believe that further use of task-based approaches in sonification research may help to ultimately ground sonifications in a more accessible - and perhaps embodied - aesthetic framework, thus leading to the development of more easily interpretable sonifications. If this is done, it could broaden the ICAD community's impact and generate wider awareness of, and interest in, the field.

\section{AKNOWLEDGMENT}

We wish to thank Dr. Masaki Matsubara at the University of Tsukuba for use of his sonification designs and MATLAB code.

\section{REFERENCES}

[1] Kramer, B. Walker, T. Bonebright, P. Cook, J. 
Flowers, N. Miner, J. Neuhoff, R. Bargar, S. Barrass, J. Berger, G. Evreinov, W. Fitch, M. Gröhn, S. Handel, H. Kaper, H. Levkowitz, S. Lodha, B. Shinn-Cunningham, M. Simoni, and S. Tipei, "Sonification Report: Status of the Field and Research Agenda," 1999.

[2] S. Bly, "Multivariate Data Mappings," in Auditory Display: Sonification, Audification, and Auditory Interfaces, G. Kramer, Ed. Westview Press, 1994, pp. 405-416.

[3] S. Barrass, "Auditory Information Design," Aust. Natl. Univ. Aust., p. 288, 1997.

[4] B. Walker, "Magnitude Estimation of Conceptual Data Dimensions for Use in Sonification," J. Exp. Psychol. Appl., vol. 8, no. 4, pp. 211-221, 2002.

[5] J. Anderson, "Creating an Empirical Framework for Sonification Design," in Proceedings of ICAD 05 Eleventh Meeting of the International Conference on Auditory Display, 2005, pp. 393-397.

[6] B. Walker and M. Nees, "Theory of Sonification," in Principles of Sonification: An Introduction to Auditory Display and Sonification, 2011, pp. 1-32.

[7] S. Barrass, "Sonification Design Patterns," Int. Conf. Audit. Disp. 2003, no. July, pp. 170-175, 2003.

[8] A. de Campo, "A Data Sonification Design Sace Map," in Proceedings of the 2nd International Workshop on Interactive Sonification, 2007, pp. 1-4.

[9] S. Barrass and P. Vickers, "Sonification Design and Aesthetics," in The Sonification Handbook, T. Hermann, A. Hunt, and J. Neuhoff, Eds. 2011, pp. 145-171.

[10] G. Leplatre and I. McGregor, "How to Tackle Auditory Interface Aesthetics? Discussion and Case Study," in Proceedings of ICAD 04 - Tenth Meeting of the International Conference on Auditory Display, 2004.

[11] C. Henkelmann, "Improving the aesthetic quality of realtime motion data sonification," Comput. Graph. Tech. Rep., pp. 1-133, 2007.

[12] G. Baier, T. Hermann, and U. Stephani, "Eventbased sonification of EEG rhythms in real time," Clin. Neurophysiol., vol. 118, no. 6, pp. 1377-1386, 2007.

[13] D. Worrall, "Parameter Mapping Sonic Articulation and the Perceiving Body," in The 16nth International Conference on Auditory Display, 2010, pp. 207-214.

[14] S. C. Peres, "A Comparison of Sound Dimensions for Auditory Graphs: Pitch Is Not So Perfect," $J$. Audio Eng. Soc., no. July, pp. 561-567, 2012.

[15] P. Vickers, C. Laing, M. Debashi, and T. Fairfax, "Sonification Aesthetics and Listening for Network Situational Awareness," SoniHED - Conf. Sonification Heal. Environ. Data, 2014.

[16] S. Pauletto and A. Hunt, "The Sonification of EMG Data," Proc. 12th Int. Conf. Audit. Disp., pp. 152 $157,2006$.

[17] D. Worrall, "A Method for Developing an Improved Mapping Model for Data Sonification," in The 17th International Conference on Auditory Display, 2011.

[18] S. Roddy and D. Furlong, "Embodied Aesthetics in Auditory Display," Organised Sound, vol. 19, no. Special Issue 01. pp. 70-77, 2014.

[19] S. Roddy and B. Bridges, "Sounding Human with Data: The Role of Embodied Conceptual Metaphors and Aesthetics in Representing and Exploring Data Sets," pp. 64-76, 2016.
[20] M. Nees and B. Walker, "Listener, Task, and Auditory Graph: Toward a Conceptual Model of Auditory Graph Comprehension," in Proceedings of the 13th International Conference on Auditory Display, 2007, pp. 266-273.

[21] B. Walker and G. Kramer, "Sonification Design and Metaphors: Comments on Walker and Kramer, ICAD 1996," ACM Trans. Appl. Percept., vol. 2, no. 4, pp. 413-417, 2005.

[22] G. Dubus and R. Bresin, "A systematic review of mapping strategies for the sonification of physical quantities," PLoS One, vol. 8, no. 12, 2013.

[23] G. Kramer, Auditory Display: Sonification, Audification, and Auditory Interfaces. Westview Press, 1994.

[24] J. Flowers, "Thirteen Years of Reflection on Auditory Graphing: Promises, Pitfalls, and Potential New Directions," in Proceedings of ICAD05 Eleventh Meeting of the International Conference on Auditory Display, 2005, pp. 406-409.

[25] P. Sanderson, J. Anderson, and M. Watson, "Extending Ecological Interface Design to Auditory Displays," in Proceedings of the 2000 Annual Conference of the Computer-Human Interaction Special Interest Group (CHISIG) of the Ergonomics Society of Australia, 2000, pp. 259-266.

[26] B. N. Walker, J. Lindsay, and J. Godfrey, "The Audio Abacus: Representing Numerical Values with Nonspeech Sound for the Visually Impaired," in ASSETS, 2004.

[27] S. C. Peres, D. Verona, T. Nisar, and P. Ritchey, "Towards a Systematic Approach to Real-Time Sonification Design for Surface Electromyography," Displays, vol. 47, pp. 25-31, 2017.

[28] D. Phipps, G. Meakin, P. Beatty, C. Nsoedo, and D. Parker, "Human Factors in Anaesthetic Practice: Insights from a Task Analysis," Br. J. Anaesth., vol. 100, no. 3, pp. 333-344, 2008.

[29] G. van der Veer, B. Lenting, and B. Bergevoet, "GTA: Groupware Task Analysis - Modeling Complexity," Acta Psychol. (Amst)., vol. 91, pp. 297-322, 1996.

[30] B. Kirwan and L. Ainsworth, Eds., A Guide to Task Analysis. CRC Press, 1992.

[31] D. Embrey, "Task Analysis Techniques," 2000. [Online]. Available: http://www.humanreliability.com/articles/Task Analysis Techniques.pdf.

[32] R. Clark, D. Feldon, J. Merrienboer, K. Yates, and S. Early, "Cognitive Task Analysis," in Handbook of research on educational communications and technology, 2008, pp. 577-593.

[33] S. Card, T. Moran, and A. Newell, The Psychology of Human-Computer Interaction. Lawrence Erlbaum Associates, 1983.

[34] B. John and D. Kieras, "The GOMS Family of User Interface Analysis Techniques: Comparison and Contrast," ACM Trans. Comput. Interact., vol. 3, no. 4, pp. 320-351, 1996.

[35] M. Matsubara, H. Terasawa, H. Kadone, K. Suzuki, and S. Makino, "Sonification of Muscular Activity in Human Movements Using the Temporal Patterns in EMG," in Signal \& Information Processing Association Annual Summit and Conference (APSIPA ASC), 2012 